\title{
Anti-CCP Antibodies: an Emerging Marker for the Early Diagnosis of Rheumatoid Arthritis
}

\author{
Sarah $\mathrm{K}$ and Nadeem $\mathrm{A}$ \\ Department of Immunology, University of Health Sciences, Lahore, Pakistan \\ ABSTRACT
}

\begin{abstract}
Rheumatoid arthritis (RA) is a systemic disease affecting mainly joints. Since rheumatoid arthritis is a common and a world wide problem therefore accurate diagnosis of this disease is essential. In the past rheumatoid factor was considered as the only marker for diagnosing this disease; however rheumatoid factor is present in many other disorders and even in up to $15 \%$ of healthy population. For the detection of rheumatoid arthritis, over the years many auto antibodies have been reported such as anti perinuclear factor (APF) and anti keratin antibodies (AKA) but they were not specific and due to the tedious procedure used for their laboratory diagnosis, they could not become popular for the diagnosis of this disease. Recently anti-CCP antibodies have been reported and they are becoming popular due to their high sensitivity and specificity. It is hoped that in a couple of years time anti-CCP antibodies could be included in the ACR criteria for diagnosing RA.
\end{abstract}

KEYWORDS: Rheumatoid factor, anti perinuclear factor, anti keratin antibodies, anti-cyclic citrullinated peptide antibodies

\section{INTRODUCTION}

Rheumatoid arthritis (RA) is an autoimmune disease of unknown etiology associated with chronic inflammation of joints and there is production of several auto antibodies that often lead to joint destruction and disability. ${ }^{1},{ }^{2}$ The prevalence of rheumatoid arthritis is about $1 \%$ world wide and is more common in women than men (2.5:1 ratio). A patient is said to have rheumatoid arthritis that has at least four of the criteria mentioned in the list of American College of Rheumatology (ACR).

Rheumatoid arthritis is a complex disease where several genetic, environmental and stochastic factors are responsible for its development. The exact etiology of the disease is still unknown but several theories have been proposed for its development. Genetic susceptibility is quite significant for the development of rheumatoid arthritis. There is a well defined familial predisposition and a high rate of prevalence for this disease in monozygotic twins. ${ }^{4}$ More than 30 years ago an association between shared epitope of HLA-DRB allele and RA was documented. ${ }^{5}$ Further, it was believed that HLA-DRB1 phenotype was not only associated with the disease but also correlated with the severity of disease as well. ${ }^{6},{ }^{7}$ HLADRB1 01 and HLADRB1 03 were found more common in Pakistani rheumatoid patients. ${ }^{8}$ Now several other genetic loci

Corresponding author;

Dr Nadeem Afzal

Head, Department of Immunology,

University of Health Sciences,

Khayaban-e-Jamia Punjab,

e-mail:aann30@hotmail.com are also found responsible for the development of this disease e.g. PTPN22, TRAFI-C5 locus etc. Interestingly, a strong association was noticed between HLADRB1 and cigarette smoking as well. ${ }^{9}$

The clinical course of RA is variable and initially there is malaise, fatigue, mucoskeletal pain which is usually followed by joints involvement. Patients with RA are more at risk to develop myocardial infarction. Seropositive rheumatoid arthritis patients with nodular manifestations also have vascular complications. There can be both intraneural and extraneural pathological processes e.g. carpal tunnel syndrome and posterior interosseous nerve palsy respectively in this disease. ${ }^{10}$ Recently some experimental studies have suggested the role of toll like receptors (TLR) signaling pathway in the pathogenesis of rheumatoid arthritis. ${ }^{11}$

Rheumatoid arthritis is associated with the production of several auto antibodies which include rheumatoid factor (RF), anti-perinuclear factor (APF), anti-neutrophil cytoplasmic antibody (ANCA), antiRA33, anti-flaggerin antibodies, anti-keratin antibodies (AKA), anti-cyclic citrullinated peptide antibodies (CCP), etc. ${ }^{12}$ Anti perinuclear factor and anti keratin antibodies are the tests known for a long time for their high specificity for rheumatoid arthritis. Both of these assays were performed by immunofluorescencs technique but these tests could not become popular because of technical difficulties and tedious procedures. ${ }^{13},{ }^{14}$ It is now evident that APF, AKA and anti-Sa antibodies targets citrullinated proteins. ${ }^{15}$

Rheumatoid factor (RF) consists of antibodies directed against Fc portion of immunoglobulin-G (IgG) and it can be detected in $60-80 \%$ of RA patients. ${ }^{16}$ RF can also be detected in other connective tissue diseases, chronic inflammatory diseases, and various infections 
such as malaria, in elderly population and even in healthy individuals. ${ }^{17}$ Although RF is not a specific test for rheumatoid arthritis yet according to ACR, determination of RF is one of the essential criteria to diagnose rheumatoid arthritis.

Anti-CCP antibodies: Citrullination is post-translational modification of protein-bound arginine into non-standard amino acid citrulline. Enzymatic conversion of arginine to citrulline is catalyzed by peptidylarginine (PAD) enzyme. $2,{ }^{18}$ Anti-CCP antibodies can react with several citrullinated peptides on multiple proteins such as flaggrin, vimentin, fibrin, and alpha enolase, therefore these antibodies are labeled as anti-citrullinated protein/peptide antibodies (ACPA). Among various auto antibodies, RF and anti-CCP antibodies are considered as useful markers for the diagnosis of RA. ${ }^{2}$

Anti-CCP antibodies were first described by Schellekens et al and they documented its high specificity (98\%) and high sensitivity (79\%) in the diagnosis of RA. ${ }^{19}$ There were similar findings by various studies in RA patients where researchers have reported $95-98 \%$ specificity of anti-CCP antibody. Anti-CCP antibody has been found to be more specific than RF and this increased specificity is an advantage of this test. ${ }^{20}$ Early diagnosis of rheumatoid arthritis is essential to minimize joint damage and these anti-CCP antibodies can be detected in the sera of patients even before the appearance of characteristic signs and symptoms of this disease. First generation anti-CCP test kits had sensitivity of $60-68 \%$ while second generation antiCCP2 test were developed with sensitivity of $75-80 \%$ and now third generation anti-CCP antibody detection kit have been developed. ${ }^{21}$

Initially, anti-CCP antibodies were associated with RF positive polyarticular patients in juvenile idiopathic arthritis (JIA) and therefore, it was suggested that these antibodies should not be investigated routinely in JIA patients. ${ }^{22}$ However, recent studies documented anti-CCP antibodies in both IgM-RF-positive and IgMRF-negative JIA children, and even at an early stage of the disease as well. ${ }^{23}$ Presence of anti-CCP antibody in the serum can be detected much earlier than the onset of RA and therefore, there is a possibility for the detection of these antibodies even in pre-clinical stage of the disease. $.^{18},{ }^{24}$ Further, anti-CCP antibodies have been detected at an early stage of the disease in seronegative rheumatoid arthritis patients as well. ${ }^{25}$ Several connective tissue diseases such as systemic lupus erythmatosus (SLE) and sjogren's syndrome (SS) can also manifest with joint involvement which make these diseases indistinguishable from RA and therefore, it increases the risk for misdiagnosis of rheumatoid arthritis. In a retrospective study, it was determined that anti-CCP could distinguish RA, SLE and other deforming arthopathies. Another study suggested that anti-CCP antibody could be better linked to RA than SLE. ${ }^{26}$ The low prevalence of anti-CCP in isolated SLE patients confirmed that anti-CCP is almost completely confined to RA. Sjogren syndrome also shares several clinical features with RA but anti-CCP antibodies were found in only $7.5 \%$ of patients with primary sjogern's syndrome as compare to RA. ${ }^{27}$

Although anti-CCP antibodies have same sensitivity as of RF yet anti-CCP is a better predictor of the course of disease over a period of 3 years and concentration of anti-CCP antibodies increases with the severity of disease. ${ }^{28},{ }^{29}$ Patients with anti-CCP antibodies developed severe disease with more radiological destruction as compared RA patients who did not have theses auto antibodies. ${ }^{30}$

A study showed smokers have increased levels of RF and are more prone to develop RA and anti-CCP antibodies were also higher in these persons. ${ }^{31}$ Probably when lung encounters smoke and other irritants, macrophages are activated and some go into apoptosis, necrosis, or both. This leads to increased synthesis and activity of peptidylarginine deaminases (PAD) enzymes, which cause citrullination in certain proteins in lungs. Some of these post-translationally modified proteins bind specifically to HLA-DR molecules on antigen-presenting cells; dendritic cells or macrophages that contain shared epitope peptide-binding motif. This process determines strength of immune response to citrullinated peptides. Smoking might further contribute to T-cell and B-cell activation by triggering antigen presenting cells in lung, thus enhancing cell-cell interactions (e.g. T cell receptor-HLA-DR, CD40Ligand-CD40, and several other events), which finally result in high titer of anti citrullinated peptide antibodies.

Recently another auto antibody i.e. anti-MCV has been reported. These antibodies are directed against citrullinated vimentin and are reactive to citrullinated proteins. The sensitivity and specificity of anti-MCV antibodies are 69.5 and $90.8 \%$ respectively as compared 70.1 and $98.7 \%$ for anti-CCP 2 assay. Therefore, due to high specificity anti-CCP is a superior test to anti-MCV for the diagnosis of rheumatoid arthritis. ${ }^{32}$ In conclusion, detection of anti-CCP antibodies is a useful diagnostic tool, particularly in the early stages of rheumatoid arthritis. ${ }^{33}$ Since a good serological marker should be sensitive, highly specific, detectable at early stage of disease and help in the prognosis of disease, therefore anti-CCP antibody due to its high specificity and sensitivity may assist in the diagnosis of RA. It is suggested that anti-CCP antibody may hold promise in becoming a key serological marker for RA as its diagnostic and prognostic value has been proved. ${ }^{34}$ Hence, anti-CCP antibodies could be an important addition to our diagnostic armamentarium. ${ }^{35}$ 


\section{REFERENCES}

1. De Rycke L, Peene I, Hoffman AEI, et al. Rheumatoid factor and anti-citrullinated protein antibodies in Rheumatoid arthritis: diagnostic value, associations with radiological progression rate, and extra-articular manifestation. Ann Rheum Dis 2004; 63:1587-93

2. Alessandri C, Priori R, Modesti M, et al. Role of anti-CCP Abs testing in Rheumatoid arthritis. Clinic Rev Allerg Immunol 2007; 34:45-9

3. Klareskog L, Catrina IA, Paget S. Rheumatoid arthritis. Lancet 2009; 373:659-72

4. Kumar V, Abbas A, Fausto N. Robbins \& Cotran Pathologic Basis of Disease. 7th ed. Philadelphia: Saunders, 2005:1273-1324

5. De Vries RP, Huizinga TWJ, Toes REM. Redefining the HLA and RA association: To be or not to be antiCCP positive. J Autoimmun 2005; 25:21-5

6. Panayi SG, Wooley P, Batchelor RJ. Genetic basis of rheumatic disease: HLA antigens, disease manifestations and toxic reactions to drugs. Br Med J 1978; 2:1326-28

7. Weyand MC, Xie C, Goronzy JJ. Homozygosity for HLA-DRB1 allele selects for extraarticular manifestations in rheumatoid arthritis. J Clin Invest 1992; 89:2033-39

8. Ali AA, Moatter T, Baig AJ, et al. Polymorphism of HLA-DR and HLA-DQ in rheumatoid arthritis patients and clinical response to methotrexate a hospital based study. J Pak Med Assoc 2006; 56:45256

9. Padyukov L, Silva C, Stolt P, et al. A gene- environment interaction between smoking and shared epitopes genes in HLA-DR provides a high risk of seropositive rheumatoid arthritis. Arthritis Rheum 2004; 50:3085-92

10. Hayton $M$. Vascular and neurological considerations in rheumatoid arthritis. Int Congr Ser 2006; 1295:34-42

11. Brentano F, Kyburz D, Schorr O, et al. The role of Toll-like receptor signaling in the pathogenesis of arthritis. Cell Immunol 2005; 233:90-6

12. Bas S, Perneger TV, Seitz M, et al. Diagnostic tests for RA Comparison of anti-cyclic citrullinated peptide antibodies, anti-keratin antibodies and $\lg M$ rheumatoid factor. Rheumatology 2002; 41:80914

13. Vossenaar RE, Venrooij VJW. Anti-CCP antibodies a highly specific marker for early rheumatoid arthritis. Clin Appl Immunol Rev 2004; 4:239-62

14. Khosla P, Shankar S, Duggal L. Anti-CCP antibodies in rheumatoid arthritis. J

Indian Rheumatol Assoc 2004; 12:143-46

15. Schellekens GA, de Jong BA, van den Hoogen, FH, et al. Citrulline is an essential constituent of antigenic determinants recognized by rheumatoid arthritis specific auto antibodies. J Clin Invest 1998; 101:273-81

16. Rheumatoid Factor. In Wikipedia [online]. Available at: http://en.wikipedia.org/wiki/ Rheumatoid_factor. Accessed April 1, 2010.

17. Lutteri L, Malaise M, Chapelle PJ. Comparison of second and third generation anti-CCP antibody assay for detecting rheumatoid arthritis. Clinica Chimica Acta 2007; 386:76-81

18. Zendman WJA, Venrooij JW, Pruijin MJG. Use and significance of anti- CCP autoantibodies in rheumatoid arthritis. Rheumatology 2006; 45:20-5

19. Schellekens GA, Visser H, de Jong BA, et al. The diagnostic properties of RA antibodies recognizing cyclic citrullinated peptide. Arthritis Rheum 2000; 43:155-63

20. Komoun M. Diagnostic performances and predictive value of anti-CCP Abs for diagnosis of RA. Clin Chem 2005; 51:12-13

21. Vallbracht I, Helmke K. Additional diagnostic and clinical value of anti-CCP Ab compared with RF Isotypes in RA. Autoimmun Rev 2005; 4:389-94

22. Brunner J, Sitzmann FC. The diagnostic value of anti-CCP Abs in children with JIA. Clin Exp Rheumatol 2006; 24:449-51

23. Lipinska J, Smolewska E, Brozik H, et al. Anti-CCP Abs in children with JIA diagnostic and clinical significance. Centr Eur J Immunol 2008; 33:19-23 
